\title{
Permeability through Cellulose Membranes Grafted with Vinyl Monomers in a Homogeneous System IV. Methyl Methacrylate Grafted Cellulose Membranes
}

\author{
Noboru Nishioka, Osamu Fujimoto, Masato TACHibana, \\ Masakuni UNO, ${ }^{*}$ and Kouichi KOSAI \\ Faculty of Engineering and ${ }^{*}$ Junior College Division, \\ Osaka Electro-Communication University, \\ Neyagawa, Osaka 572, Japan
}

(Received March 18, 1987)

\begin{abstract}
The homogeneous grafting of methyl methacrylate onto cellulose was carried out in a dimethyl sulfoxide (DMSO)-paraformaldehyde solvent system. Three kinds of membranes were prepared: membranes of graft copolymers using ammonium persulfate (APS) as an initiator (APS membranes), membranes of those using azobisisobutyronitrile, and blended membranes of cellulose and poly(methyl methacrylate). The diffusive permeabilities of solutes through the membranes, the states of water in them, and their microphase separated structures depended on the kind of membrane. The permeabilities of solutes through three kinds of membranes were inferior to those through the membrane cast from the DMSO solution of cellulose. The APS membranes indicated specificity of permeation for vitamine $B_{12}$, poly(ethylene glycol), and water. It was found that the fine microphase separated structure is essential for the improvement of the permeability through the membranes.
\end{abstract}

KEY WORDS Cellulose / Methyl Methacrylate / Graft Membrane / Diffusive Permeability / Microphase Separated Structure / States of Water / Freezing Water / Nonfreezing Water / Activation Energy for Permeation /

The diffusive permeation of solutes through water-swollen membranes is considered to occur through water phases in them. ${ }^{1-7}$ But the different states of water are present in membranes. $^{8-19}$ Thus, characterization and quantification of water relating to the permeation of solutes seem significant for clarifying the mechanism of membrane permselectivity.

In membranes of different affinity for water, prepared from amphiphilic copolymers of various compositions, the states of water will differ from one another. In our previous studies, ${ }^{20-22}$ cellulose grafted with vinyl monomers in a homogeneous system was used as membrane materials. The permeabilities of solutes through the copolymer membranes and the states of water in them were investigated. The use of acrylonitrile (AN) and methyl acrylate (MA) as hydrophobic vinyl monomers decreased the water content of the membranes but improved the permeabilities of solutes through them. This was due to the change in the states of water in the membranes and increase in freezing water content.

In this study, homogeneous grafting onto cellulose was carried out with methyl methacrylate (MMA) which is thought more hydrophobic than AN and MA. The diffusive permeabilities of solutes through the MMA grafted cellulose membranes of various compositions are investigated in relation to the composition, microphase separated structures, and states of water in them. 


\section{EXPERIMENTAL}

\section{Grafting and Membrane Preparation}

The homogeneous grafting of MMA onto cellulose in the dimethyl sulfoxide (DMSO)paraformaldehyde solvent system and characterization of the graft products were performed by the method described in our previous papers. $^{23,24}$

The homogeneous symmetrical membranes were prepared by the same method as that described previously. ${ }^{20}$ The crude grafting mixtures were cast at room temperature on glass plates, and DMSO and unreacted MMA were allowed to evaporate at about $40^{\circ} \mathrm{C}$ under reduced pressure for $24 \mathrm{~h}$ prior to the coagulation of membranes in water. The DMSO solutions of cellulose and poly(methyl methacrylate) (PMMA) were mixed to give proper blended ratios. The blended membranes were also prepared by the method described above.

\section{Diffusive Permeability}

The diffusive permeability coefficients, $P$, were measured at given temperatures from 20.0 to $50.0^{\circ} \mathrm{C}$ by the method described in our previous paper. ${ }^{20}$ Eight solutes of different molecular weights were used. The viscosityaverage molecular weights, $\bar{M}_{v}$, for commercial poly(ethylene glycol) (PEG) samples were estimated from the intrinsic viscosities, [ $\eta]$, obtained with benzene at $25.0^{\circ} \mathrm{C}:{ }^{25} \mathrm{PEG}-\mathrm{IV}=$ $3.1 \times 10^{3}, \mathrm{PEG}-\mathrm{VI}=8.0 \times 10^{3}$, and PEG-XX= $3.1 \times 10^{4}$, respectively.

\section{Hydraulic Permeability}

The hydraulic permeability of water was measured with a reverse osmosis batch cell (Fuji Seiki FMD300) having a volume of $300 \mathrm{~cm}^{3}$ and an effective membrane area of $24.6 \mathrm{~cm}^{2}$.

\section{Transmission Electron Microscopy (TEM)}

A Hitachi HU-11A transmission electron microscope was used to observe the micro- phase separated structures of the membranes. The membranes were stained by immersion in a $2 \%$ aqueous solution of osmic acid for one day.

\section{Differential Scanning Calorimetry (DSC)}

The measurements were performed with SEIKO SSC/560 DSC according to the method described previously. ${ }^{21}$ The samples were cooled with liquid nitrogen at a rate of $5^{\circ} \mathrm{C} \mathrm{min}^{-1}$ to $-80^{\circ} \mathrm{C}$ and then heated at the same rate to $60-90^{\circ} \mathrm{C}$. The water content was varied by standing the sample pan in the DSC cell at a given temperature for a given period to evaporate the water.

\section{RESULTS AND DISCUSSION}

The three kinds of membranes used are characterized in Table I. The MMA grafted cellulose membranes were classified into two groups depending upon the grafting initiator used, i.e., ammonium persulfate (APS) or azobisisobutyronitrile (AIBN). It is known that the difference in initiator influences the grafting efficiency (GE) expressing the ratio of weight of graft polymer to that of polymer formed and that the use of AIBN leads to less GE because of the resonance stabilization of its radical fragments. ${ }^{27}$ In the $\mathrm{AN}$ and MA grafted cellulose membranes, ${ }^{20,22}$ the difference in GE was remarkable. But it has been reported $^{23,24,28}$ that the chain transfer reactivity of PMMA macroradicals to cellulose is higher than those of PAN and PMA macroradicals. Thus, even for the AIBN membranes, the values of GE were around $50 \%$ in the MMA grafted cellulose membranes.

Copolymers indicate microphase separated structures due to the incompatibility of constituent polymers. ${ }^{29}$ The samples used in this study contain the homopolymer component. Therefore, the microphase separated structures depended not only on the total PMMA content but also on the homo-PMMA content. Figure 1 shows the TEM micrographs of the 
Permeability through MMA Grafted Cellulose Membranes

Table I. Characterization of grafted and blended membranes

\begin{tabular}{|c|c|c|c|c|c|c|}
\hline \multirow{2}{*}{ Sample code } & \multirow{2}{*}{$\frac{G E}{\%}$} & \multicolumn{3}{|c|}{ PMMA content $/ \%$} & \multirow{2}{*}{$\begin{array}{c}\bar{M}_{v} \times 10^{-4} \\
\text { of } \text { graft }^{\mathrm{a}}\end{array}$} & \multirow{2}{*}{$\begin{array}{l}\text { Number } \\
\text { of grafts }\end{array}$} \\
\hline & & Overall & Graft-PMMA & Homo-PMMA & & \\
\hline \multicolumn{7}{|l|}{ APS membrane } \\
\hline$g$-PMMA 1 & 81.8 & 5.9 & 4.8 & 1.1 & 11.2 & 0.06 \\
\hline$g$-PMMA 2 & 63.2 & 17.4 & 11.0 & 6.4 & 11.5 & 0.19 \\
\hline$g$-PMMA 3 & 96.0 & 29.2 & 28.0 & 1.2 & 6.2 & 1.14 \\
\hline \multicolumn{7}{|l|}{ AIBN membrane } \\
\hline$g$-PMMA 11 & 60.8 & 30.4 & 18.4 & 12.0 & 7.8 & 0.54 \\
\hline$g$-PMMA 12 & 54.2 & 45.6 & 24.7 & 20.9 & 9.0 & 0.81 \\
\hline$g$-PMMA 13 & 41.9 & 53.3 & 22.3 & 31.0 & 5.3 & 1.44 \\
\hline$g$-РMMA 14 & 6.7 & 61.4 & 4.1 & 57.3 & 5.2 & 0.33 \\
\hline \multicolumn{7}{|c|}{ Blended membrane ${ }^{b}$} \\
\hline$b$-PMMA 1 & 0 & 10.0 & 0 & 10.0 & & \\
\hline$b$-PMMA 2 & 0 & 20.0 & 0 & 20.0 & & \\
\hline$b$-PMMA 3 & 0 & 30.0 & 0 & 30.0 & & \\
\hline$b$-PMMA 4 & 0 & 40.0 & 0 & 40.0 & & \\
\hline$b$-PMMA 5 & 0 & 50.0 & 0 & 50.0 & & \\
\hline
\end{tabular}

a $\bar{M}_{r}$ was estimated from $[\eta]$ obtained in acetone at $25.0 \mathrm{C}^{26}$

c $\bar{M}_{r}$ for cellulose and PMMA are $16 \times 10^{4}$ and $16 \times 10^{4}$, respectively. ${ }^{23.26}$
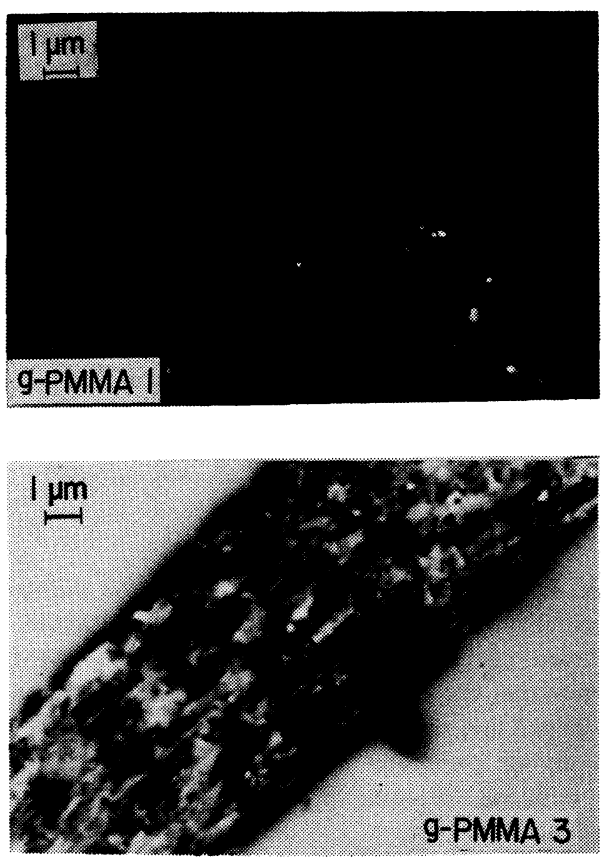

Figure 1. Transmission electron micrographs of APS membranes stained with osmic acid.
APS membranes. The dark domains indicate the cellulose phases stained with osmic acid. The microphase separated structures are very fine and the interfaces between cellulose and PMMA phases are not clear. The TEM micrographs of the AIBN membranes are shown in Figure 2. The Ellipsoidal domains with dark surroundings and white spherical domains are dispersed in the gray or white matrix. The interfaces between both domains are clearer than those in the APS membranes. In Figure 3, the TEM micrographs of the blended membranes are indicated. The white PMMA domains are dispersed in the cellulose matrix. The $b$-PMMA 5 membrane with higher PMMA content indicates larger white domains. The difference in microphase separated structures for three kinds of membranes is remarkable. This has also been found for the AN and MA grafted cellulose membranes. ${ }^{20.22}$ The microphase separated structures for AN, MA, and MMA grafted cellulose membranes differ from each other, suggesting the difference in the compatibility of each vinyl polymer 

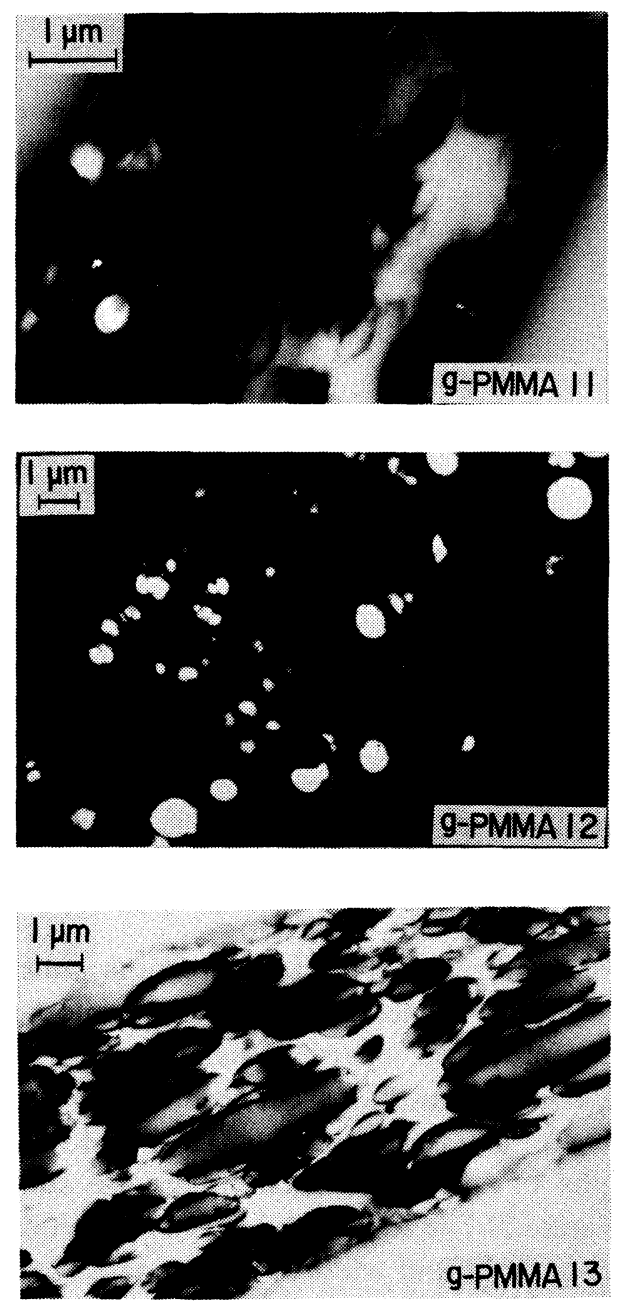

Figure 2. Transmission electron micrographs of AIBN membranes.

with cellulose.

Figure 4 demonstrates the solute molecular weight dependence of permeability for various membranes. The results of our previous study for the commercial regenerated cellulose membrane, Cuprophan, and the membrane cast from the DMSO solution of cellulose, designated as the cellulose membrane, are also indicated. ${ }^{20}$ The permeability through each membrane decreases with increasing molecular weight of the permeant. The permeability of vitamine $\mathrm{B}_{12}$ through the $g$-PMMA 3 mem-
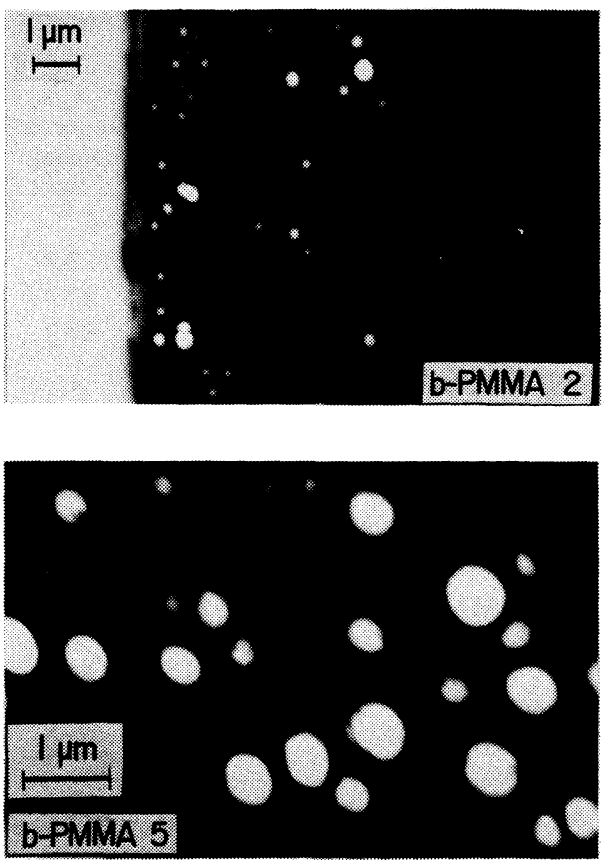

Figure 3. Transmission electron micrographs of the blended membranes.

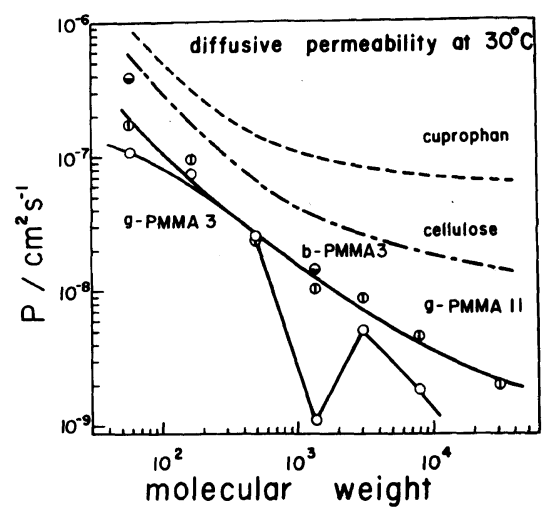

Figure 4. Molecular weight dependence of diffusive permeability for various membranes: (O) $g$-PMMA 3 membrane; (1) $g$-PMMA 11 membrane; ( $\ominus$ ) $b$-PMMA 3 membrane; the broken line, Cuprophan membrane; dash-dotted line, cellulose membrane; the solutes are, in the order of increasing molecular weight, $\mathrm{NaCl}$, uric acid, raffinose, vitamine $B_{12}$, PEG-IV, PEG-VI, and PEG-XX.

brane, an APS membrane, is very low. Moreover, the permeabilities of two PEG samples are also low and in particular PEG-XX 
hardly permeated through this membrane. On the other hand, the permeability through the $g$ PMMA 11 membrane, an AIBN membrane, is inferior to that through the cellulosic membranes, but the molecular weight dependence for these membranes is similar. There is also no peculiarity of the permeability of vitamine $\mathrm{B}_{12}$ through the $b$-PMMA 3 membrane, a blended membrane. As can be seen from Table I, the PMMA content of these three membranes is about $30 \%$, but the grafting efficiency differs from each other.

Figures 5 and 6 reveal the relationship between PMMA content and permeability for various solutes. The data for the Cuprophan and cellulose membranes are also indicated. The permeabilities of solutes through the APS and AIBN membranes decrease slightly with increasing PMMA content. The permeabilities of lower molecular weight solutes, i.e., $\mathrm{NaCl}$, uric acid, and raffinose, through both kinds of membranes are almost on the same level. But there exists clearly the difference in the permeability of vitamine $B_{12}$. Moreover, the permeabilities of PEG samples through the AIBN membranes are superior to those through the APS membranes. The inferiority of the permeability of PEG through the APS membranes to that through the cellulosic membranes has also been found for the AN and MA grafted cellulose membranes, ${ }^{20,22}$ but not so remarkable as that for the MMA grafted cellulose membranes. The permeability of $\mathrm{NaCl}$ through the blended membranes is superior to that through other two kinds of membranes, but the permeability of vitamine $B_{12}$ through the blended membranes is almost the same as that through the AIBN membranes. It is clear that the permeability through the membranes depends on the permeating solute itself. In the $\mathrm{AN}$ and MA grafted cellulose membranes, the permeability through the APS membranes increased with increasing vinyl polymer content and leveled off, but that through the AIBN and blended membranes decreased. ${ }^{20,22}$ These results,

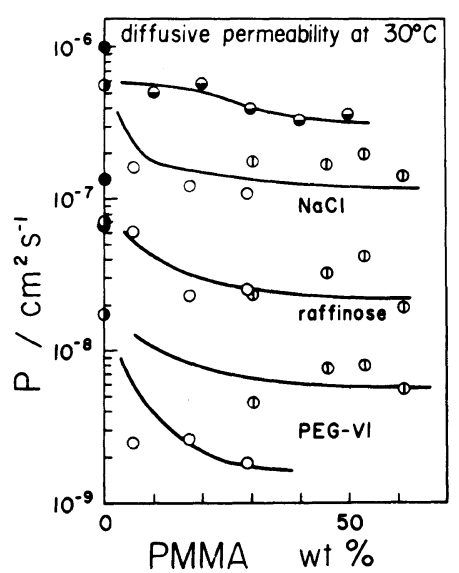

Figure 5. PMMA content dependence of diffusive permeability for $\mathrm{NaCl}$, raffinose, and PEG-VI: (O) Cuprophan membrane; (O) cellulose membrane; (O) APS membranes; (D) AIBN membranes; (Ө) Blended membranes.

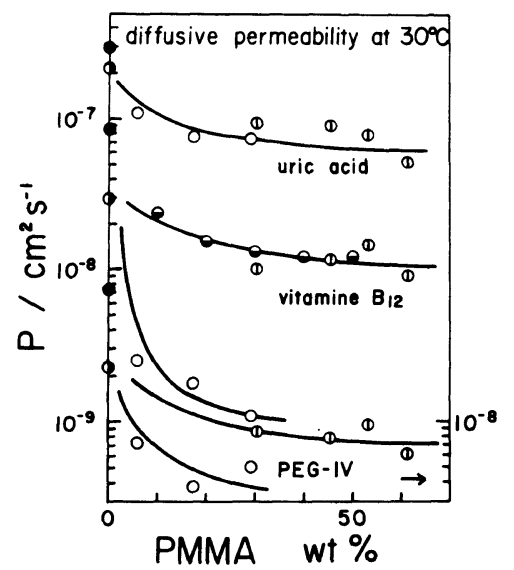

Figure 6. PMMA content dependence of diffusive permeability for uric acid, vitamine $B_{12}$, and PEG-IV; the symbols are the same as those in Figure 5.

therefore, indicate that the permeability through the membranes depends on the vinyl polymer incorporated; that is, a specific interaction between solutes and membrane substrates must be taken into account.

The freezing and melting thermograms for the cellulosic and MMA grafted cellulose membranes are drawn in Figures 7 and 8. Several peaks are found on both thermograms. The peaks at higher and lower temperatures 


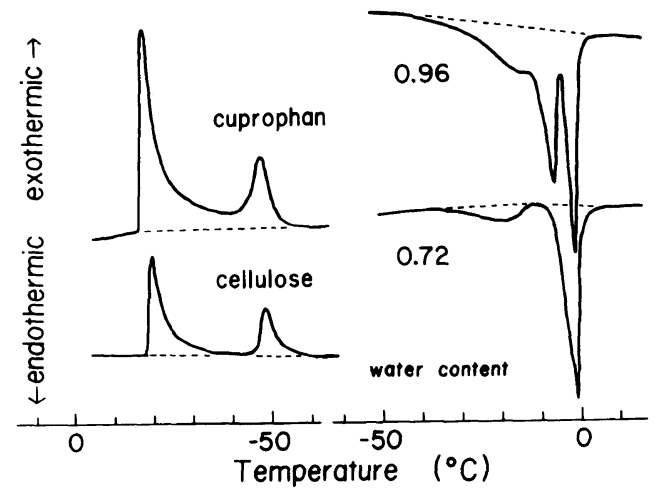

Figure 7. DSC freezing and melting curves for the Cuprophan and cellulose membranes.

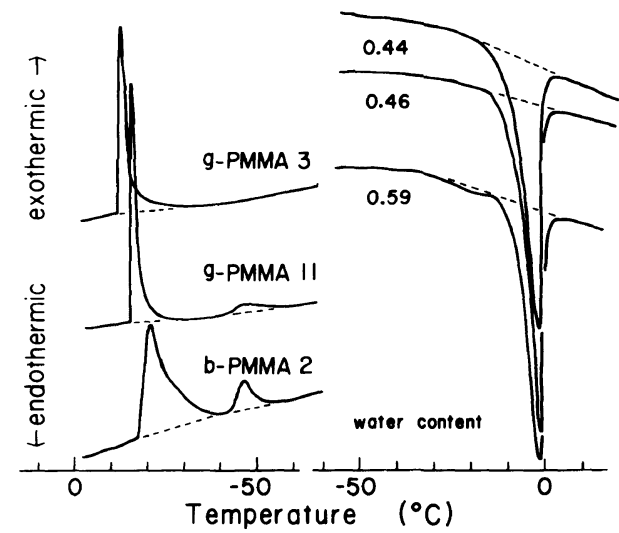

Figure 8. DSC freezing and melting curves for the MMA grafted and PMMA blended cellulose membranes.

were assigned to free water and water interacting weakly with polymer molecules, i.e., intermediate water, respectively. ${ }^{21}$ The thermograms for the MMA grafted cellulose membranes are different from those for the cellulosic membranes. In the $g$-PMMA 3 membrane, the exothermic peak at around $-50^{\circ} \mathrm{C}$ which is observed for the cellulosic membranes disappears. Furthermore, neither are the endothermic peaks at around -20 and $-10^{\circ} \mathrm{C}$ recognized. Similar thermographic features were observed for the $g$-PMMA 3 membrane of different water contents and the other APS membranes.

The small exothermic peak at around $-50^{\circ} \mathrm{C}$ is recognized for the $g$-PMMA 11 membrane, but the fraction of this peak is smaller than those of the cellulosic membranes. The endothermic curve of the $g$ PMMA 11 membrane shows a similar peak as that of the $g$-PMMA 3 membrane. Such a difference in the number of peaks between freezing and melting thermograms was also observed for the AN grafted cellulose membranes, ${ }^{21}$ implying that the observation of both thermograms is important to understand the states of water in membranes more precisely.

On the other hand, both thermograms of the $b$-PMMA 2 membrane are similar to those of the cellulose membrane. However, the tailing of exothermic free water peak is more remarkable than that of the cellulose membrane. Such tailing was characteristic of the PAN and PMA blended cellulose membranes. ${ }^{21.22} \mathrm{~A}$ comparison of Figure 8 with Figure 7 reveals that the incorporation of hydrophobic PMMA into hydrophilic cellulose influenced the states of water in the membranes, especially the states of intermediate water, and exothermic peak temperature for free water. The degree of influence decreases in the order of APS, AIBN, and blended membranes. This means that the finer the microphase separated structures of the membranes, the more the states of water in them are influenced.

The shift of peak temperature accompanying the change in water content is depicted in Figure 9. Each peak temperature shifts toward lower temperatures with decreasing water content and exothermic peak temperature changes sharply at around 0.5 water content. The explanation for this is that the water molecules interacting more weakly with polymer molecules decrease faster with decreasing water content than those interacting more strongly. $8,13,17,18,21,22$

Figure 10 illustrates the relationship between water content and heat of melting of freezing water, $\Delta H$, determined from the area under the endothermic curve. The data points 
Table II. Amounts of water in cellulosic, grafted, and blended membranes

\begin{tabular}{|c|c|c|c|}
\hline \multirow{3}{*}{ Sample code } & $\begin{array}{l}\text { Equilibrium } \\
\text { water content }\end{array}$ & $\begin{array}{c}\text { Equilibrium nonfreezing } \\
\text { water content }\end{array}$ & $\begin{array}{l}\text { Equilibrium freezing } \\
\text { water content }\end{array}$ \\
\hline & $W_{\mathrm{DSC}}$ & $W_{\text {non }}$ & $W_{\mathrm{DSC}}-W_{\text {non }}$ \\
\hline & \multicolumn{3}{|c|}{ g- $\mathrm{H}_{2} \mathrm{O} /$ g-dry membrane } \\
\hline \multicolumn{4}{|c|}{ Cellulosic membrane } \\
\hline Cuprophan & 1.00 & 0.49 & 0.51 \\
\hline Cellulose & 0.70 & 0.45 & 0.25 \\
\hline \multicolumn{4}{|l|}{ APS membrane } \\
\hline$g$-PMMA 1 & 0.47 & 0.30 & 0.17 \\
\hline$g$-PMMA 2 & 0.47 & 0.33 & 0.14 \\
\hline$g$-PMMA 3 & 0.42 & 0.28 & 0.14 \\
\hline \multicolumn{4}{|l|}{ AIBN membrane } \\
\hline$g$-PMMA 11 & 0.45 & 0.29 & 0.16 \\
\hline$g$-РMMA 13 & 0.35 & 0.21 & 0.14 \\
\hline$g$-PMMA 14 & 0.32 & 0.19 & 0.13 \\
\hline \multicolumn{4}{|l|}{ Blended membrane } \\
\hline$b$-PMMA 2 & 0.57 & 0.39 & 0.18 \\
\hline$b$-PMMA 5 & 0.37 & 0.22 & 0.15 \\
\hline
\end{tabular}

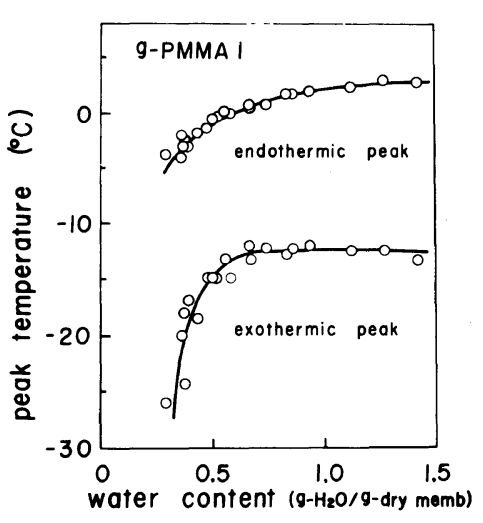

Figure 9. Effects of water content on endothermic peak and exothermic peak temperatures.

for each membrane do not follow a straight line but give a curve convexing downward at lower water content. The slope of each straight part of the curve is approximately equal to the specific heat of melting of pure water. A similar result has also been reported for other membranes; ${ }^{8.9 .21 .22}$ it is thus considered that the nonfreezing water is not saturated at lower water contents. The equilibrium nonfreezing

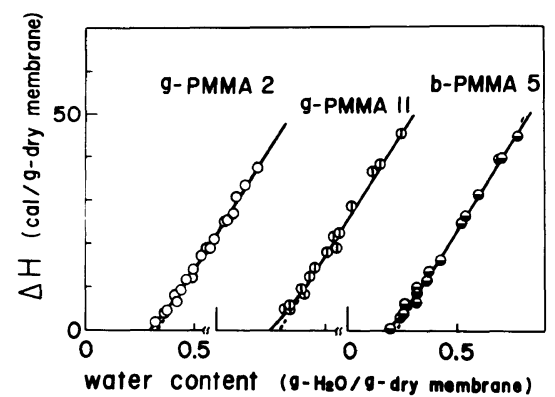

Figure 10. Total water content dependence of the heat of melting of freezing water for various membranes: $(O)$ $g$-PMMA 2 membrane; (1) $g$-PMMA 11 membrane; (๑) $b$-PMMA 5 membrane.

water content, $W_{\text {non }}$, was estimated by extrapolation of the straight part of the curve to $\Delta H=0$. The results are summarized in Table II. As mentioned in our previous papers, ${ }^{20.21}$ the equilibrium water content determined by blotting water on the membrane surfaces involved a considerable experimental error. It has been found ${ }^{22}$ that there exists good correlation between the water content where the curve changes sharply in Figure 9 and the 
Table III. Apparent activation energy for permeation of solutes and hydraulic permeation of water

\begin{tabular}{lcccc}
\hline & \multicolumn{4}{c}{ Apparent activation energy } \\
\cline { 2 - 4 } Solute & \multicolumn{2}{c}{$\mathrm{kcal} \mathrm{mol}^{-1}$} \\
& Cuprophan & Cellulose & g-PMMA 2 & g-PMMA 11 \\
\cline { 2 - 4 } & 4.3 & 4.2 & 4.9 & 3.8 \\
$\mathrm{NaCl}$ & 3.8 & 4.3 & 5.5 & 4.2 \\
Urea & 4.6 & 4.8 & 5.3 & 4.9 \\
Uric acid & 4.6 & 5.1 & 7.1 & 5.3 \\
Raffinose & 4.4 & 5.0 & 10.8 & 4.8 \\
Vitamine $\mathrm{B}_{12}$ & 3.2 & 3.2 & 7.6 & 3.4 \\
PEG-VI & 4.2 & 3.6 & 10.8 & 3.6 \\
$\mathrm{H}_{2} \mathrm{O}^{\mathrm{a}}$ & & & & \\
\hline
\end{tabular}

a Hydraulic permeation of water.

content where the curve deviates from a straight line in Figure 10. Thus, in this study, their average value was also used as the equilibrium water content estimated by the DSC method, $W_{\text {DSC. }}$ The results are listed in Table II. The equilibrium freezing water content was calculated from the difference in the amounts of $W_{\text {DSC }}$ and $W_{\text {non }}$.

The values of $W_{\text {DSC }}$ and $W_{\text {non }}$ for each membrane decrease with increasing PMMA content, indicating the influence of the incorporation of hydrophobic PMMA. On the other hand, the freezing water content thought to be related to the permeation of solutes also decreases slightly with increasing PMMA content, being consistent with the results shown in Figures 5 and 6 . The amounts of freezing water for the cellulosic membranes are larger than those for the three kinds of membranes, explaining the superiority of the cellulosic membranes shown in Figure 4. This is inconsistent with our previous results, ${ }^{21.22}$ since the affinity for water in the MMA grafted cellulose membranes became too hydrophobic to increase the freezing water content. It is difficult to elucidate the specificity of permeability through the APS membranes by means of differences in freezing water content, suggesting that the freezing water content is not the only measure of solute permeability. It has been reported that some solutes can permeate the nonfreezing water regions $\mathrm{s}^{8,30}$ and that solutes having water structure breaking hydrophilic groups are closely accessible to polymer molecules. ${ }^{12,15,31,32}$ In addition to the clarification of the detail of freezing water, partition of solute between different states of water, and permeation through nonfreezing water region, an interaction between solute and membrane substrate must be taken into account in order to elucidate the permselectivity through membranes.

Apparent activation energies for permeation of solutes through the membranes, determined from the Arrhenius plot, are listed in Table III. Activation energy is thought to be a measure indicating the ease of solute permeation or the extent of an interaction between solute and membrane substrate. Activation energies for permeation of solutes through the cellulosic membranes scarcely depend on the permeating solutes. Although the molecular weight of PEG-VI is highest within these solutes, the activation energy for it is lowest, implying the difference in the permeation mechanism for PEG. Activation energies through the $g$ PMMA 11 membrane are almost the same as those through the cellulosic membranes, elucidating a similar molecular weight dependence of permeability through these three mem- 
branes. Activation energies through the $g$ PMMA 2 membrane are a little larger than those through the cellulosic membranes. Especially, activation energies for vitamine $\mathrm{B}_{12}$ and PEG-VI are a few times larger than those through the cellulosic membranes. This is consistent with the inferiority of permeabilities of these solutes through the $g$-PMMA 2 membrane to those through the cellulosic membranes and means the difference in the permeation mechanism for these solutes or the specific interaction between the MMA grafted cellulose membranes and the solutes.

Activation energies for hydraulic permeation of water through the cellulosic and $g$ PMMA 11 membranes are similar to that for purely viscous flow of water through a rigid capillary. ${ }^{33}$ But activation energy through the $g$-PMMA 2 membrane is larger than those through other membranes. This indicates the difference in the permeation mechanism not only for vitamine $\mathrm{B}_{12}$ and PEG but for water. It has been reported ${ }^{34.35}$ that there exists hydrophobic hydration water in the vicinity of hydrophobic polymers and that hydrophobic polymers facilitate the clustering tendency of water to lead a decrease in solute solubility. The specificity of permeability through the APS membranes may be associated with hydrophobic hydration water. It is of interest that only the APS membranes with fine microphase separated structures indicate such specificity of permeation. In the AN and MA grafted cellulose membranes, improved permeability also occurred for the APS membranes. $^{20.22}$ It should be emphasized that the fine microphase separated structures in the membranes are significant. In other words, it is important that the hydrophobic polymer chains around the hydrophilic cellulose chains influence the states of water in the membranes.

\section{REFERENCES}

1. H. Yasuda, C. E. Lamaze, and L. D. Ikenberry, Makromol. Chem., 118, 19 (1968).
2. H. Yasuda, L. D. Ikenberry, and C. E. Lamaze, Makromol. Chem., 125, 108 (1969).

3. H. Yasuda, A. Peterlin, C. K. Colton, K. A. Smith, and E. W. Merrill, Makromol. Chem., 126, 177 (1969).

4. J. Komiyama and R. M. Fuoss, Proc. Natl. Acad. Sci., U.S.A., 69, 829 (1972).

5. S. Takigami, Y. Maeda, and Y. Nakamura, J. Appl. Polym. Sci., 24, 1419 (1979).

6. S. Takigami, M. Nakamura, and Y. Nakamura, Polymer, 25, 963 (1984).

7. Y. Kojima, K. Furuhata, and K. Miyasaka, J. Appl. Polym. Sci., 29, 533 (1984).

8. Y. Taniguchi and S. Horigome, J. Appl. Polym. Sci., 19, 2743 (1975).

9. R. A. Nelson, J. Appl. Polym. Sci., 21, 645 (1977).

10. M. F. Froix and R. Nelson, Macromolecules, 8, 726 (1975).

11. D. G. Pedley and B. J. Tighe, Br. Polym. J., 11, 130 (1979)

12. S. J. Wisniewski and S. W. Kim, J. Membrane Sci., 6, 299 (1980).

13. C. Lemoyne, C. Friedrich, J. L. Halary, C. Noël, and L. Monnerie, J. Appl. Polym. Sci., 25, 1883 (1980).

14. Y. Ikada, M. Suzuki, and H. Iwata, in "Water in Polymers," S. P. Rowland, Ed., American Chemical Society, Washington D.C., 1980, p 287.

15. S. C. Yoon and M. S. Jhon, J. Appl. Polym. Sci., 27, 3133 (1982).

16. I. D. Maxwell and R. A. Pethrick, J. Appl. Polym. Sci., 28, 2363 (1983).

17. H. Ohno, M. Shibayama, and E. Tsuchida, Makromol. Chem., 184, 1017 (1983).

18. K. Nakamura, T. Hatakeyama, and H. Hatakeyama, Polymer, 24, 871 (1983).

19. A. Higuchi and T. Iijima, Polymer, 26, 1207 (1985).

20. N. Nishioka, K. Watase, K. Arimura, K. Kosai, and M. Uno, Polym. J., 16, 867 (1984).

21. N. Nishioka, S. Yoshimi, T. Iwaguchi, and K. Kosai, Polym. J., 16, 877 (1984).

22. N. Nishioka, T. Kuromatsu, T. Takahashi, M. Uno, and K. Kosai, Polym. J., 18, 131 (1986).

23. N. Nishioka and K. Kosai, Polym. J., 13, 1125 (1981)

24. N. Nishioka, K. Matsumoto, and K. Kosai, Polym. J., 15, 153 (1983).

25. G. Allen, C. Booth, S. J. Hurst, M. H. Hones, and C. Price, Polymer, 8, 391 (1967).

26. H. J. Cantow and G. V. Schulz, Z. Physik. Chem., 2, 117 (1954).

27. F. Ide, "Graft Jugo to Sono Oyo," Kobunshi Kankokai, Kyoto, 1977.

28. N. Nishioka, K. Minami, and K. Kosai, Polym. J., 15, 591 (1983).

29. S. Kraus, in "Polymer Blends," Vol. 1, D. R. Paul and S. Newman, Ed., Academic Press, New York, N.Y., 1978, p 15. 
30. A. Higuchi and T. Iijima, J. Appl. Polym. Sci., 32, 3229 (1986).

31. E. G. Finer, F. Franks, and M. J. Tait, J. Am. Chem. Soc., 94, 4424 (1972).

32. O. D. Bonner, J. M. Bednarek, and R. K. Arisman, J. Am. Chem. Soc., 99, 2898 (1977).

33. R. A. Horne, "Water and Aqueous Solutions,"
Wiley, New York, N.Y., 1972.

34. T. Kinoshita, T. Tanigami, S. Washizu, S. Hayashi, A. Takizawa, and Y. Tsujita, Polym. J., 12, 475 (1980).

35. A. Takizawa, T. Kinoshita, O. Nomura, and Y. Tsujita, Polym. J., 17, 747 (1985). 